\title{
Ontology semantic approach to extraction of knowledge from Holy Quran
}

\begin{abstract}
With the continued demand for Islamic knowledge, which is mainly based on the Quran as a source of knowledge and wisdom, systems that facilitate an easy search of the content of the Quran remain a considerable challenge. Although in recent years there have been tools for Quran search, most of these tools are based on keyword search, meaning that the user needs to know the correct keywords before being able to retrieve the content of al-Quran. In this paper, we propose a system that supports the end user in querying and exploring the Quran ontology. The system comprises user query reformulation against the Quran ontology stored and annotated in the knowledge base. The Quran ontology consists of noun concepts identified in al-Quran, and the relationship that exists between these concepts. The user writes a query in the natural language and the proposed system reformulates the query to match the content found in the knowledge base in order to retrieve the relevant answer. The answer is represented by the Quranic verse related to the user query.
\end{abstract}

Keyword: Islamic knowledge; Keyword matching; Ontology; Semantic technology 